\title{
The gliadin-CFTR connection: new perspectives for the treatment of celiac disease
}

\author{
Luigi Maiuri ${ }^{1,2^{\wedge}}$ D, Valeria R. Villella ${ }^{1}$, Valeria Raia ${ }^{3}$ and Guido Kroemer ${ }^{4,5,6,7,8,9^{*}}$
}

\begin{abstract}
Familial loss-of-function mutations of the gene coding for the cystic fibrosis transmembrane conductance regulator (CFTR) channel protein cause cystic fibrosis (CF), the most frequent inherited life-threatening disease in the Caucasian population. A recent study indicates that the gluten/gliadin-derived peptide (P31-43) can cause CFTR inhibition in intestinal epithelial cells, thus causing a local stress response that contributes to the immunopathology of celiac disease (CD). Accordingly, an increased prevalence of CD has been observed in several cohorts of CF patients. CD is characterized by a permanent intolerance to gluten/gliadin proteins occurring in a proportion of susceptible individuals who bear the human leukocyte antigen (HLA) DQ2/DQ8. In CD, perturbations of the intestinal environment, together with the activation of the innate immune system by P31-43, are essential for rendering other immunodominant gliadin peptide fully antigenic, thus triggering an adaptive immune response with an autoimmune component. P31-43induced CFTR inhibition elicits the danger signals that ignite the epithelial stress response and perturb epithelial proteostasis. Importantly, potentiators of CFTR channel gating, such as the FDA-approved drug Ivacaftor, prevent P31-43 driven CFTR inhibition and suppress the gliadin-induced stress response in cells from celiac patients, as well as the immunopathology developing in gliadin-sensitive mice. Thus, CFTR potentiators may represent a novel therapeutic option for celiac patients.
\end{abstract}

Keywords: CFTR, Celiac disease, Gliadin

\section{Background}

The intestinal mucosa constitutes the first-line defense against dietary or microbial challenges and usually avoids unwarranted inflammatory reactions in response to nonself-antigens by promoting oral tolerance [1]. The capacity of the mucosal immune system to neutralize harmful luminal challenges, can be subverted by exogenous triggers, such as viruses [2], or conditions in which still undefined inherited or acquired cell-autonomous factors favor an intestinal pro-inflammatory state. Cystic fibrosis (CF), the most frequent monogenic lethal disease in the Caucasian population [3], is the quintessential example of a disease in which cell-autonomous triggers favor antigen mishandling by the intestinal mucosa. Indeed, in the CF intestine, two

\footnotetext{
*Correspondence: kroemer@orange.fr

'Deceased

${ }^{4}$ Equipe1 1 labellisée Ligue Nationale contrele Cancer, Centre de Recherche des Cordeliers, Paris, France

5 INSERM U1138, Centre de Recherche des Cordeliers, Paris, France

Full list of author information is available at the end of the article
}

unfavorable events determine an inadequate cellular and humoral immune response to food components, (i) the increased antigenic load due to pancreatic insufficiency and (ii) the constitutive chronic intestinal inflammation due to loss-of function-mutations in the $\mathrm{CF}$ transmembrane conductance regulator (CFTR) gene $[4,5]$. Accordingly, CF patients often manifest increased levels of antibodies against alimentary antigens, including anti-gliadin IgA antibodies, increased intestinal permeability, elevated levels of fecal calprotectin, shifts in the intestinal microbiota, and increased intestinal permeability [5-7]. Importantly, CF patients manifest a threefold increase in the prevalence of celiac disease $(\mathrm{CD})[8,9]$ a permanent intolerance to gluten/gliadin proteins that occurs in a proportion of susceptible individuals who bear the human leukocyte antigen (HLA) DQ2/ DQ8 [10-12]. Of note, a prevalence as high as $\sim 4 \%$ of positive anti-TG2-IgA autoantibodies, a serological marker of $\mathrm{CD}$, has been reported in several cohorts of CF patients [5-7], even in the absence of villous damage, the hallmark

(c) The Author(s). 2019 Open Access This article is distributed under the terms of the Creative Commons Attribution 4.0 International License (http://creativecommons.org/licenses/by/4.0/), which permits unrestricted use, distribution, and 
of $\mathrm{CD}$. Thus, there is an epidemiological link between $\mathrm{CF}$ and $\mathrm{CD}$.

\section{$\mathrm{CF}$ and $\mathrm{CF}$, a mysterious connection}

The unexpected link between $\mathrm{CF}$ and $\mathrm{CD}$, the gluten enteropathy triggered the hypothesis that CFTR might be involved in gluten sensitivity. Mounting evidence supports this hypothesis. Indeed, CFTR is not only an anion channel relevant to $\mathrm{CF}$, but is also a hub protein that orchestrates the proteostasis network of epithelial cells, including enterocytes, thus regulating adaptation to cell-autonomous or external stress [13-19]. Loss of CFTR function causes increased generation of reactive oxygen species (ROS) and persistent activation of tissue transglutaminase (TGM2) [13-19], which targets several TGM2 substrates, including the autophagy-relevant Beclin 1 protein (BECN1), hence suppressing autophagy. Moreover, BECN1 targeting by TGM2 results in the functional sequestration of BECN\&asssociated phosphatidylinositol 3-kinase catalytic subunit type 3 (PIK3C3) with reduced availability of the PIK3C3 product phosphatidyl-inositol-3-phosphate (PtdIns3P) at early endosomes, thus impairing endosomal maturation and trafficking $[13,14]$.

In addition, TGM2 activation leads to increased nuclear translocation of nuclear factor kappa-light-chain-enhancer of activated $B$ cells (NF- $\mathrm{KB}$ ) owing to TGM2 targeting of the NF- $k B$ inhibitor alpha (NFKBIA) $[13,15,18]$. NF- $k B$ activation then leads to increased levels of pro-inflam matory cytokines, including interleukin (IL)-17A, IL-21 and IL-15, a master cytokine involved in gut homeostasis [2022] as well as IL-1 $\beta$ (downstream of both NFKB and the NLRP3 inflammasome).

Interestingly, these consequences of CFTR malfunction are reminiscent of those induced by some gliadin fractions in celiac intestine [12, 23, 24]. Indeed, after gluten ingestion, two major peptides, the 25-mer and the 33-mer, remain undigested and induce innate immunity activation and adaptive Th1-mediated immune responses, respectively. Some peptide fractions, such as P31-43, a fragment of to 25-mer, are capable of triggering an enterocyte stress response that is accompanied by TGM2 activation, derangement of endosomal trafficking, increased NF-kB nuclear translocation and consequent IL-15 upregulation [10, 12, 23-26].

In the intestine from $\mathrm{CD}$ patients, the perturbation of the local environment with activation of the innate immune response by the 25-mer (or its fragment P31-43), is required to allow the immune-dominant gliadin peptides (33-mer or its fragment P57-68) to elicit a Th1 and antibody responses [10, 12, 23-26]. However, how P31-43 can induce an epithelial stress response has remained elusive, although putative additional factors as reovirus [2] or yet-to-be defined cell-autonomous events are required to provide the danger signal that can ignite P31-43 induced epithelial stress $[10,12,25]$.

\section{CFTR, an unforeseen surface receptor for gliadin} Given the consequences of CFTR malfunction observed in CF, the intriguing question arises as to whether CFTR inhibition represents the cell-autonomous event that mediates gliadin-induced stress response in $\mathrm{CD}$.

In a recent study [27] we demonstrated a molecular and functional interaction between P31-43 and CFTR. By using a multifaceted experimental approach including computer-assisted calculations, plasmon surface resonance, co-immunoprecipitation, fluorescent confocal microscopy and electrophysiological measurements of CFTR dependent chloride currents, we demonstrated that P31-43 binds to, and reduces the ATPase activity of, the nuclear binding domain 1 (NBD1) of CFTR, thus impairing CFTR channel function. The CFTR inhibitory effect of gliadin was confirmed in three different mouse models of gliadin-sensitivity [27].

CFTR oscillates between the open and closed conformational states of the chloride channel [4, 28]. Notably, P31-43 can only efficiently bind to NBD1 when it is in the closed state, leading to the block of its gating function [27]. Moreover, increasing the probability of CFTR channel opening by means of pharmacological "potentiators" of CFTR channel gating prevent CFTR inhibition by P31-43, as well as the P31-45 induced enterocyte stress response [27]. This could have clinical relevance as a CFTR potentiator, VX-770 (Ivacaftor, Kalydeko) is already FDA-approved for the treatment of CF patients bearing CFTR mutations with gating defects.

\section{CFTR, a sensor of stress}

As mentioned before, CFTR inhibition drives the activation of TGM2, a pivotal enzyme in CD pathogenesis as it deamidates P57-68, thus dramatically increasing its antigenicity [12, 23, 24, 29]. Moreover, activated TGM2 engages in multiple vicious cycles that aggravate the disease, in thus far that it crosslinks the complex formed by CFTR and P31-43, thus creating a trimolecular complex (CFTR, P31-43, TGM2) that renders CFTR inhibition irreversible. Moreover, TGM2 inactivates BECN1, thereby inhibiting autophagy, interfering with endosomal maturation and trafficking and driving inflammasome activation and IL-15 production [27].

Of note, Ivacaftor, as well as another CFTR potentiator, Vrx-532, are highly effective in preventing all these P31-43/gliadin driven manifestations. Importantly, the protective effect of Ivacaftor on intestinal epithelial cells, was abrogated when CFTR was genetically depleted, thus confirming that Ivacaftor protects enterocytes from the detrimental effects of gliadin through potentiating CFTR channel function [27]. 


\section{CFTR inhibition orchestrates the pathogenic response to gliadin}

In vivo experiments, in which 3 different mouse models of gliadin sensitivity (including non-obese diabetic (NOD) mice) were treated with Ivacaftor and orally challenged with gliadin, revealed that Ivacaftor is highly effective in preventing gliadin-induced IL-15 upregulation, inflammasome activation, increase of intestinal permeability. Notably, Ivacaftor prevents the upregulation of IL-17, IL-21, IFN- $\gamma$ induced by gliadin exposure in the small intestine of gliadin-sensitive mice and, instead, increases the production of TGF- $\beta$ and IL-10 [27].

These pre-clinical results suggest that CFTR potentiators might be a possible therapeutic strategy in CD. However, translating preclinical data into clinical application requires prior validation in an appropriate disease context. To address this issue, peripheral blood mononuclear cells (PBMC) freshly collected from celiac individuals were cocultured with intestinal epithelial cells to mimic a mucosal environment. Importantly, Ivacaftor prevented the production of IFN- $\gamma$ induced by gliadin exposure while increasing the production of IL-10. Notably, such a preventive effect of Ivacaftor against gliadin-induced immunopathology could be correlated with its ability to potentiate CFTR function [27].

Altogether these findings demonstrate that gliadin-induced CFTR malfunction is at the apex of the pathogenic cascade leading to $\mathrm{CD}[27,30]$. Indeed, Ivacaftor-mediated maintenance of CFTR function was able to avoid the all gliadin induced manifestations of $\mathrm{CD}$, both in mouse models of gluten enterophagy and in cells freshly collected from celiac individuals [27].

\section{Conclusion}

Although at present the gluten-free diet is the sole option to avoid gluten related morbidity and prevent gluten-associated pathologies, other strategies are emerging. These still experimental approaches focus on enzymes that can degrade pathogenic gliadin peptides, thus reducing gliadin "toxicity", attempt to prevent the intestinal permeabilization induced by gliadin, or aim at desensitizing celiac patients by means of "tolerogenic" vaccines [31-35]. In our recent work, we demonstrate a primordial pathogenic role for CFTR inhibition in $C D$. This discovery may open novel perspectives for the cure of celiac patients, suggesting that pharmacological stimulation of CFTR may interfere with a decisive step in the pathogenesis of $\mathrm{CD}$. It remains to be evaluated whether CFTR potentiators may be used for the treatment of $C D$ or whether they should rather be used for $\mathrm{CD}$ prevention. Moreover, it will be important to develop CFTR-potentiating agents that are less expensive than the drugs used for the treatment of CF. Thus, further efforts are required to identify natural compounds, repurposed drugs or new chemical entities endowed with the ability of potentiate CFTR channel gating.

\section{Abbreviations \\ BECN1: Beclin 1; CD: Celiac disease; CF: Cystic Fibrosis; CFTR: Cystic fibrosis transmembrane conductance regulator; FDA: Food and Drug Administration; HLA: Human leukocyte antigen; IFN-ү: Interferon gamma; IL-15: Interleukin- 15; IL-17A: Interleukin 17A; IL-21: Interleukin 21; NBD1: Nuclear binding domain 1; NF-kB: Nuclear factor kappa B; NOD mice: Non-obese diabetic mice; ROS: Reactive oxygen species; TGM2: Tissue transglutaminase 2; VX- 770: Ivacaftor}

\section{Acknowledgements}

Not applicable.

Funding

No funding.

No financial relationship relevant to this article to disclose.

Availability of data and materials

Unpublished experimental data are not included in this review.

Authors' contributions

LM, VRV, VR, and GK wrote the review. All Authors read and approved the final manuscript.

Ethics approval and consent to participate

Not applicable.

Consent for publication

Not applicable.

\section{Competing interests}

The authors declare that they have no competing interests. A patent application by LM is pending (filing date, July 26, 2017. No

102017000085714). GK is a scientific co-founder of Samsara therapeutics.

\section{Publisher's Note}

Springer Nature remains neutral with regard to jurisdictional claims in published maps and institutional affiliations.

\section{Author details}

${ }^{1}$ Department of Health Sciences, University of Eastern Piedmont, Novara, Italy. 'European Institute for Research in Cystic Fibrosis, San Raffaele Scientific Institute, Milan, Italy. ${ }^{3}$ Regional Cystic Fibrosis Center, Pediatric Unit, Department of Translational Medical Sciences, Federico II University Naples, Naples, Italy. ${ }^{4}$ Equipe 11 labellisée Ligue Nationale contrele Cancer, Centre de Recherche des Cordeliers, Paris, France. ${ }^{5}$ INSERM U1138, Centre de Recherche des Cordeliers, Paris, France. ${ }^{6}$ Université Paris Descartes, Paris, France.

${ }^{7}$ Metabolomics and Cell Biology Platforms, Institut Gustave Roussy, Villejuif, France. ${ }^{8}$ Pôle de Biologie, Hôpital Européen Georges Pompidou, AP-HP, Paris, France. 'Karolinska Institute, Department of Women's and Children's Health, Karolinska University Hospital, 17176 Stockholm, Sweden.

Received: 12 February 2019 Accepted: 27 February 2019

Published online: 21 March 2019

\section{References}

1. Kim KS, Hong SW, Han D, Yi J, Jung J, Yang BG, Lee JY, Lee M, Surh CD. Dietary antigens limit mucosal immunity by inducing regulatory $T$ cells in the small intestine. Science. 2016;351:858-63.

2. Bouziat R, Hinterleitner R, Brown JJ, Stencel-Baerenwald JE, Ikizler M, Mayassi $T$, et al. Reovirus infection triggers inflammatory responses to dietary antigens and development of celiac disease. Science. 2017;356:44-50.

3. Cutting GR. Cystic fibrosis genetics: from molecular understanding to clinical application. Nat Rev Genet. 2015;16:45-56.

4. Gadsby DC, Vergani P, Csanady L. The ABC protein turned chloride channel whose failure causes cystic fibrosis. Nature. 2006;440:477-83.

5. Ooi CY, Durie PR. Cystic fibrosis from the gastroenterologist's perspective. Nat Rev Gastroenterol Hepatol. 2016;13:175-85. 
6. Raia V, Maiuri L, de Ritis G, de Vizia B, Vacca L, Conte R, et al. Evidence of chronic inflammation in morphologically normal small intestine of cystic fibrosis patients. Pediatr Res. 2000;47(3):344-50.

7. Troncone R, Santamaria F, Ercolini P, Raia V, Panza G, de Ritis G. Increased serum antibody levels to dietary antigens in cystic fibrosis. Acta Paediatr. 1994:83:440-1.

8. Fluge G, Olesen HV, Gilljam M, Meyer P, Pressler T, Storrösten OT, et al. Comorbidity of cystic fibrosis and celiac disease in Scandinavian cystic fibrosis patients. J Cyst Fibros. 2009;8:198-202.

9. Walkowiak JA, Blask-Osipa LA, Oralewska B, Pogorzelski A, Cichy W, Sapiejka $E$, et al. Cystic fibrosis is a risk factor for celiac disease. Acta Biochim Pol. 2010;57:115-8.

10. Meresse B, Malamut G, Cerf-Bensussan N. Celiac disease: an immunological jigsaw. Immunity. 2012;36:907-19.

11. Sollid LM, Jabri B. Triggers and drivers of autoimmunity: lessons from coeliac disease. Nat Rev Immunol. 2013;13:294-302.

12. Meresse B, Ripoche J, Heyman M, Cerf-Bensussan N. Celiac disease: from oral tolerance to intestinal inflammation, autoimmunity and lymphomagenesis. Mucosal Immunol. 2009;2:8-23.

13. Luciani A, Villella VR, Esposito S, Brunetti-Pierri N, Medina D, Settembre C, Gavina $M$, et al. Defective CFTR induces aggresome formation and lung inflammation in cystic fibrosis through ROS-mediated autophagy inhibition. Nat Cell Biol. 2010;12:863-75.

14. Villella VR, Esposito S, Bruscia EM, Vicinanza M, Cenci S, Guido S, et al. Disease-relevant proteostasis regulation of cystic fibrosis transmembrane conductance regulator. Cell Death Differ. 2013;20:1101-15.

15. Villella VR, Esposito S, Bruscia EM, Maiuri MC, Raia V, Kroemer G, Maiuri L. Targeting the intracellular environment in cystic fibrosis: restoring autophagy as a novel strategy to circumvent the CFTR defect. Front Pharmacol. 2013:4:1-9.

16. Ferrari E, Monzani R, Villella VR, Esposito S, Saluzzo F, Rossin F, et al. Cysteamine re-establishes the clearance of Pseudomonas aeruginosa by macrophages bearing the cystic fibrosis-relevant F508del-CFTR mutation. Cell Death Dis. 2017;8:e2544.

17. Gavina M, Luciani A, Villella VR, Esposito S, Ferrari E, Bressani I, et al. Nebulized hyaluronan ameliorates lung inflammation in cystic fibrosis mice. Pediatr Pulmonol. 2013;48(8):761-71.

18. Romani L, Oikonomou V, Moretti S, lannitti RG, D'Adamo MC, Villella VR, et al. Thymosin a1 represents a potential potent single-molecule-based therapy for cystic fibrosis. Nat Med. 2017;23:590-600.

19. Rossin F, Villella VR, D'Eletto M, Farrace MG, Esposito S, Ferrari E, et al. TG2 regulates the heat-shock response by the post-translational modification of HSF1. EMBO Rep. 2018;19(7). pii):e45067.

20. Nichols DP, Chmiel JF. Inflammation and its genesis in cystic fibrosis. Pediatr Pulmonol. 2015;50(Suppl 40):S39-56.

21. Stone KP, Kastin AJ, Pan W. NFkB is an unexpected major mediator of interleukin-15 signaling in cerebral endothelia. Cell Physiol Biochem. 2011; 28:115-24.

22. Jabri B, Abadie V. IL-15 functions as a danger signal to regulate tissueresident T cells and tissue destruction. Nat Rev Immunol. 2015;15:771-83.

23. Maiuri L, Ciacci C, Ricciardelli I, Vacca L, Raia V, Auricchio S, et al. Association between innate response to gliadin and activation of pathogenic T cells in coeliac disease. Lancet. 2003:362:30-7.

24. Barone MV, Troncone R, Auricchio S. Gliadin peptides as triggers of the proliferative and stress/innate immune response of the celiac small intestinal mucosa. Int J Mol Sci. 2014;15:20518-37.

25. Setty M, Discepolo V, Abadie V, Kamhawi S, Mayassi T, Kent A, et al. Distinct and synergistic contributions of epithelial stress and adaptive immunity to functions of intraepithelial killer cells and active celiac disease. Gastroenterology. 2015;149:681-91 e10.

26. Cerf-Bensussan N, Meresse B. Coeliac disease \& gluten sensitivity: epithelial stress enters the dance in coeliac disease. Nat Rev Gastroenterol Hepatol. 2015;12:491-7

27. Villella VR, Venerando A, Cozza G, Esposito S, Ferrari E, Monzani R, et al A pathogenic role for cystic fibrosis transmembrane conductance regulator in celiac disease. EMBO J 2019; 38(2). pii: e100101.

28. Sheppard DN, Welsh MJ. Structure and function of the CFTR chloride channel. Physiol Rev 1999; 79(1 Suppl): S23-45.

29. Silano M, Vincentini O, Luciani A, Felli C, Caserta S, Esposito S, et al. Early tissue transglutaminase-mediated response underlies K562(S)-cell gliadindependent agglutination. Pediatr Res. 2012;71(5):532-8.
30. Maiuri L, Villella V, Piacentini M, Raia V, Kroemer G. Defective proteostasis in celiac disease as a new therapeutic target. Cell Death Dis. in press 2019.

31. Lerner A. New therapeutic strategies for celiac disease. Autoimmun Rev. 2010;9(3):144-7.

32. Veeraraghavan G, Leffler DA, Kaswala DH, Mukherjee R. Celiac disease 2015 update: new therapies. Expert Rev Gastroenterol Hepatol. 2015;9(7):913-27.

33. Khaleghi S, Ju JM, Lamba A, Murray JA. The potential utility of tight junction regulation in celiac disease: focus on larazotide acetate. Ther Adv Gastroenterol. 2016;9(1):37-46.

34. Di Sabatino A, Lenti MV, Corazza GR, Gianfrani C. Vaccine immunotherapy for celiac disease. Front Med (Lausanne). 2018;5:187.

35. Bakshi A, Stephen S, Borum ML, Doman DB. Emerging therapeutic options for celiac disease: potential alternatives to a gluten-free diet. Gastroenterol Hepatol (NY). 2012;8(9):582-8
Ready to submit your research? Choose BMC and benefit from:

- fast, convenient online submission

- thorough peer review by experienced researchers in your field

- rapid publication on acceptance

- support for research data, including large and complex data types

- gold Open Access which fosters wider collaboration and increased citations

- maximum visibility for your research: over $100 \mathrm{M}$ website views per year

At $\mathrm{BMC}$, research is always in progress.

Learn more biomedcentral.com/submissions 